\title{
LOW DISPERSION SPECTROPHOTOMETRY OF LATE TYPE STARS
}

\author{
H. MORENO \\ Departamento de Astronomía, Universidad de Chile
}

\begin{abstract}
Low dispersion (slit $60 \AA$ wide) spectrophotometric observations of late type stars have been made. The sample of stars observed cover from K0 to M8 and luminosity classes V to $\mathrm{I}$.

A selection of these stars including each luminosity class and spectral type is being analyzed. The observed intensity distributions have been corrected for atmospheric extinction and have been normalized to a common maximum intensity. The resulting instrumental intensity distributions are being studied for the best parameters for spectral classification.
\end{abstract}

The object of this communication is to report on some observations and preliminary results concerning $\mathrm{K}$ and $\mathrm{M}$ stars. The observations were made at the Cerro Tololo Inter-American Observatory using a spectrum scanner with a slit $60 \AA$ wide, attached to the $36-$ and 60 -in. telescopes. The scanner uses a plane grating blazed for $3800 \AA$ in the first order and a refrigerated 1P21 photomultiplier. The spectrum scans cover from 3000-6000 A. Each spectrum was scanned twice in succession in opposite directions of wavelength, in order to eliminate systematic errors (Gutiérrez-Moreno et al., 1966). A complete observation in both directions takes $24 \mathrm{~min}$ and it is registered in a Brown recorder with a speed of one inch per minute and a reciprocal dispersion of $10 \AA \mathrm{mm}^{-1}$.

Each night, conditions permitting, at least one pair of standard stars was observed twice. These observations allow accurate determination of atmospheric extinction as well as the reduction of the data to a uniform system. Sky scans were obtained when necessary. The observations include 92 stars up to $m_{v}=9.0$. The distribution of these stars in spectral types and luminosity classes is given in Table I. Most of the stars were observed on three nights.

No complete determinations of energy distributions have been made up to the moment. It has been preferred to make a preliminary analysis of a sample of stars covering all the MK types observed, for determining which are the points worth mea-

\section{TABLE I}

Stars observed

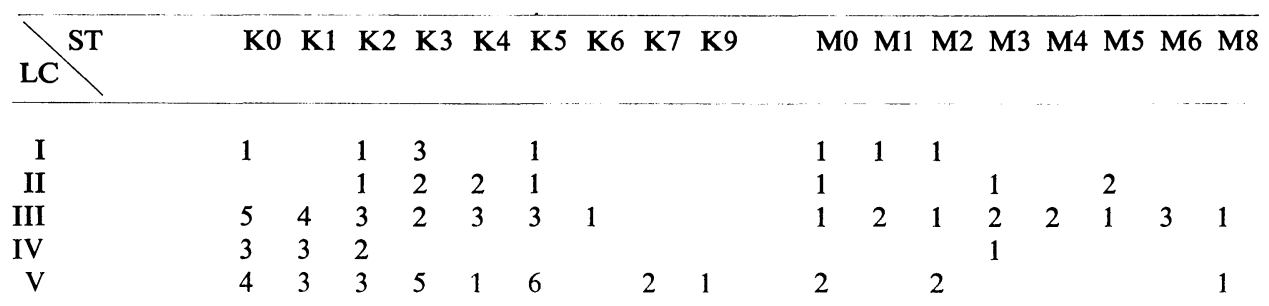

Remark: the spectral types were taken from the La Plata Catalogue (Jaschek et al., 1964). 
suring for later use in spectral classification. The instrumental energy distributions observed have been corrected for atmospheric extinction using mean values for the observing period. Amplification differences were eliminated from each spectrum and the resulting distributions were normalized to equal maximum intensity, to make the comparisons easier. The spectra of the faintest stars have lost some detail due to the large fluctuations of the signal.

Figures 1 to 4 show the variation of the energy distributions with spectral type and with luminosity class for $\mathrm{K}$ and $\mathrm{M}$ stars. Some identifications of absorption lines and bands have been made in the spectra. The identifications do not include all the lines present in a given blend, but only the most important ones. Some of the features already used for spectral classification are readily recognized in the curves. These include the $\mathrm{CN}$ bands at $3855 \AA$ and $4180 \AA$; the $\mathrm{G}$ band at $4308 \AA$; the $\mathrm{Ca}$ I line at $4226 \AA$; the $\mathrm{K}$ line of $\mathrm{Ca}$ II at $3934 \AA$; and, for the M stars, the TiO bands.

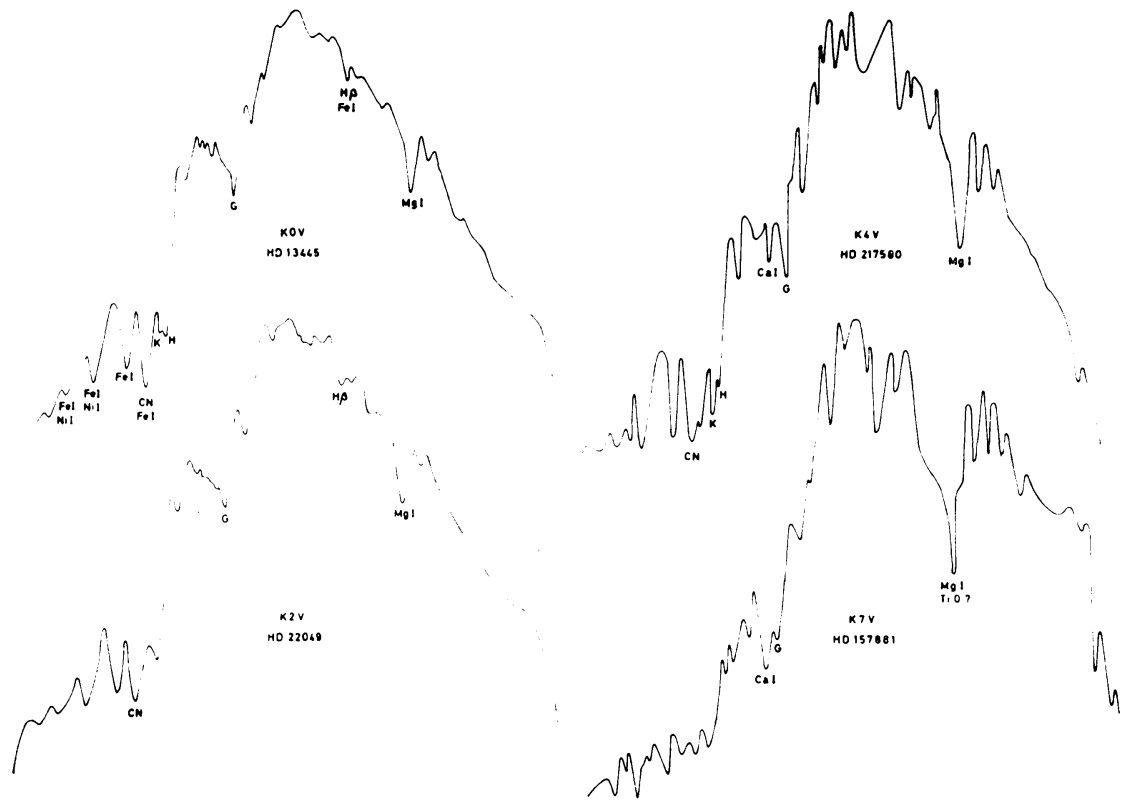

Fig. 1.

Classifications based on the schemes previously used by different authors (Lindblad and Stenquist, 1936; Elvius, 1956; Westerlund, 1951-1953; Strömgren and Gyldenkerne, 1955) have been attempted. Nevertheless, several difficulties were found, being the most important one the blends produced by the low resolution of the spectra. This makes difficult to decide what has to be measured. For example, if we measure an 'equivalent width' or the depth of a feature with respect to the apparent continuum, it is difficult to decide which continuum must be used, since this continuum keeps changing with varying spectral type or luminosity class due to the change of 


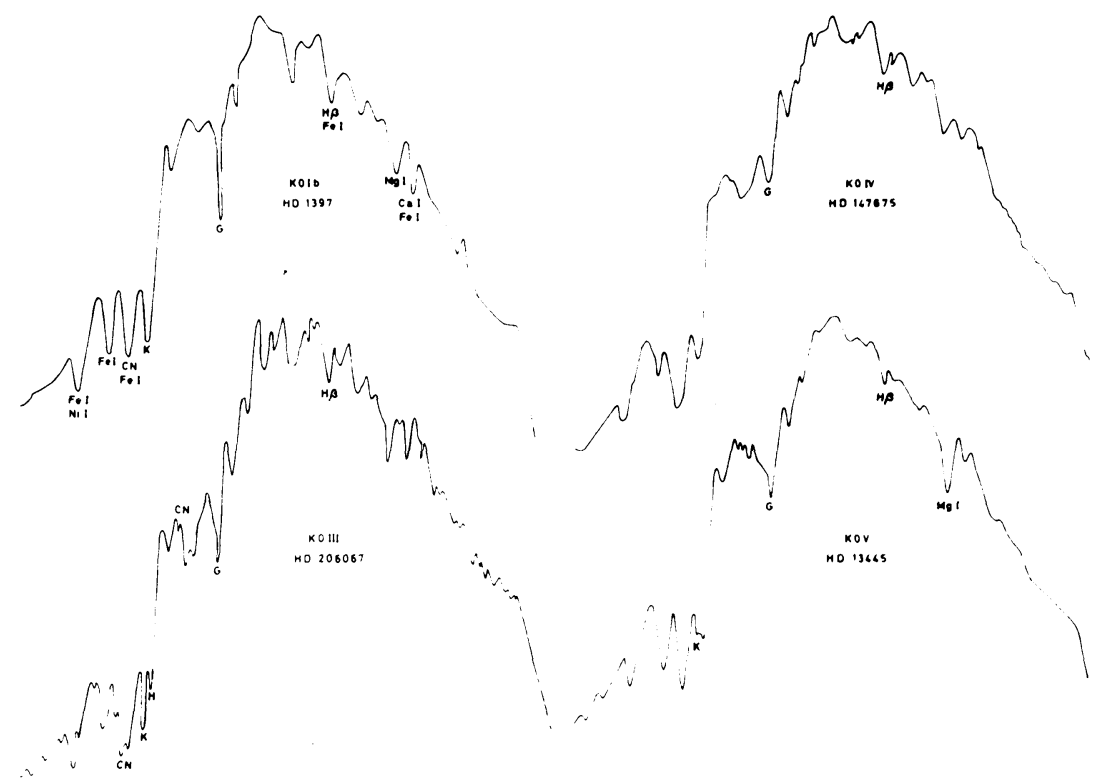

Fig. 2.

relative importance of the lines which contribute to the blend. It seems that the best results are obtained by using just the ratio of the intensities or the magnitude difference between two fixed points of the feature.

As an example, Figure 5 shows a preliminary classification scheme for $M$ stars. The $\mathrm{TiO}$ band at $4955 \AA$ has been used as a spectral type indicator, and the CaI line at $4226 \AA$ as a luminosity indicator. The indices used are:

$$
\begin{aligned}
& T=I_{4945} / I_{5020} \\
& C=I_{4190} / I_{4226}
\end{aligned}
$$

The M8V star has been classified by McCuskey (1949) using objective prism spectra. The spectrum scans suggest an earlier type.

Future work considers observations of as many stars as possible with a uniform MK classification, to fill in the gaps in Table I and to obtain homogeneous classification schemes. A computer program is being prepared for the accurate determination of intensity distributions. It includes the determination of atmospheric extinction using two approximations and considering its variation in the ultraviolet (Mendoza et al., 1968). For the program stars, the computer program includes the determination of the outside-of-the-atmosphere intensity distributions, the reduction to a uniform system and the computation of the parameters selected for classification.

The possibility of studying peculiarities, such as differences of metal contents is being considered. 


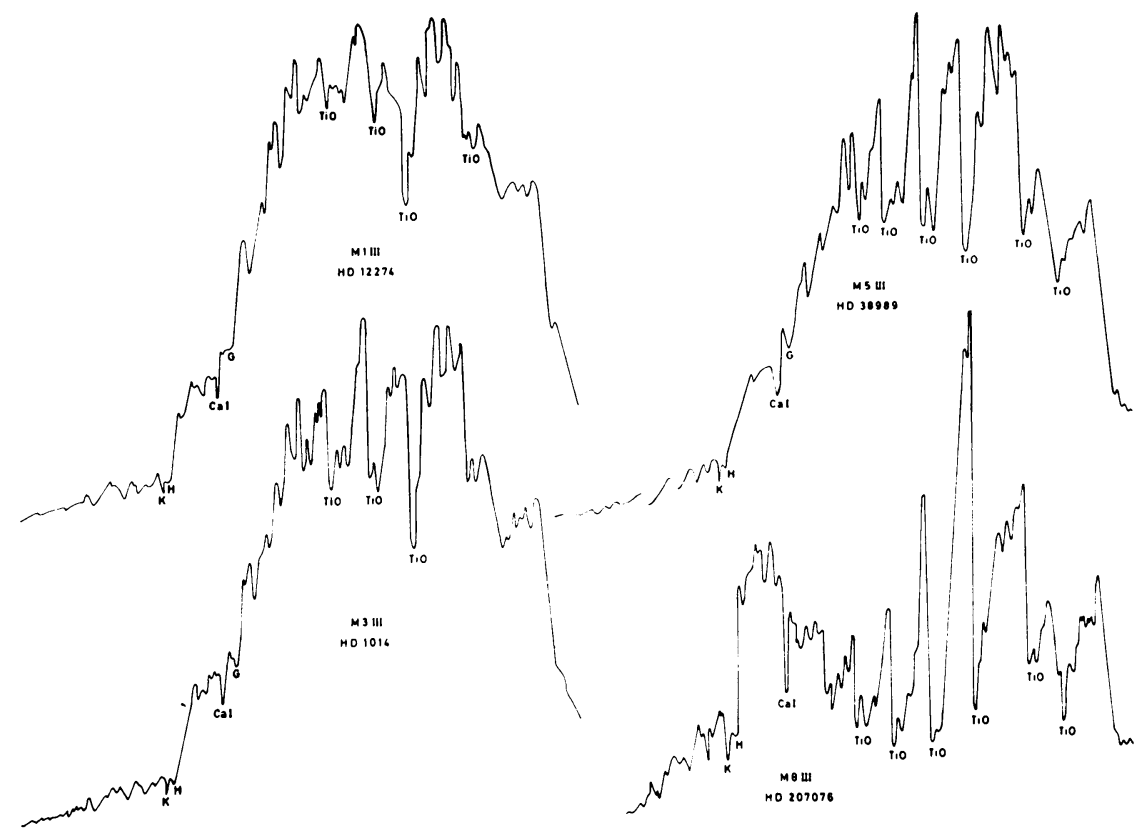

Fig. 3.

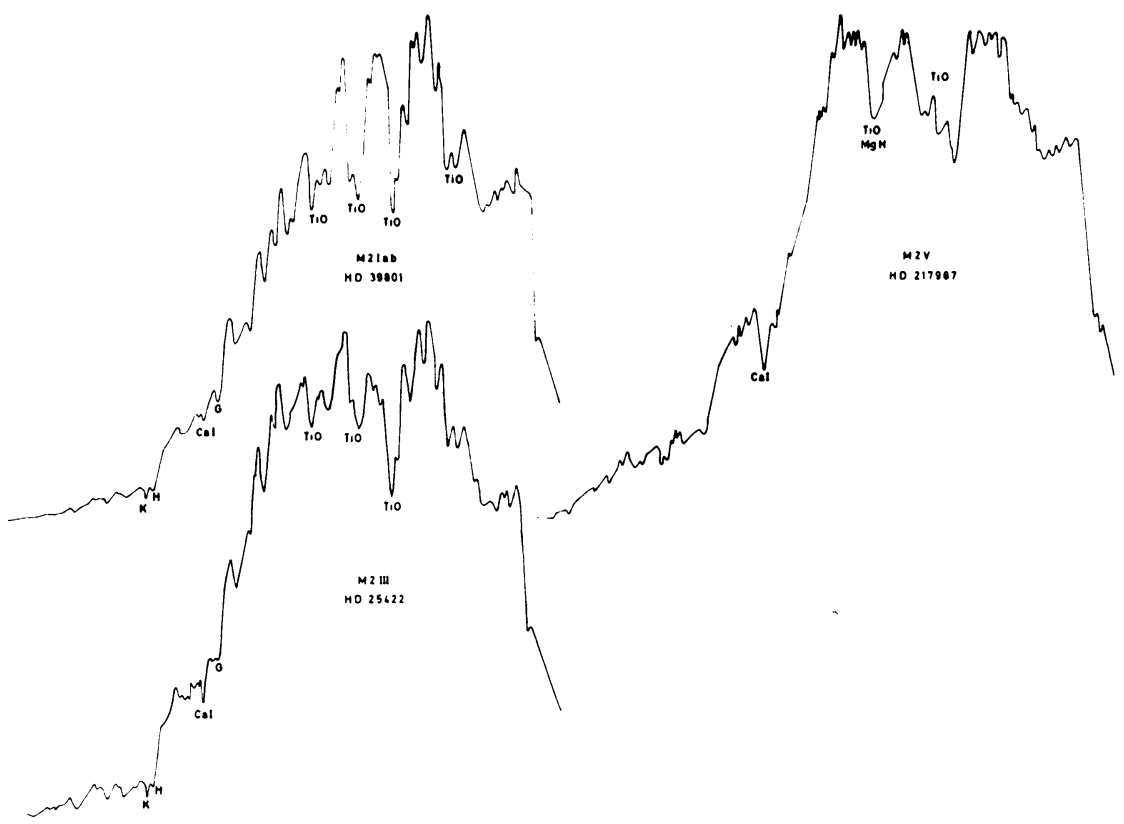

Fig. 4. 


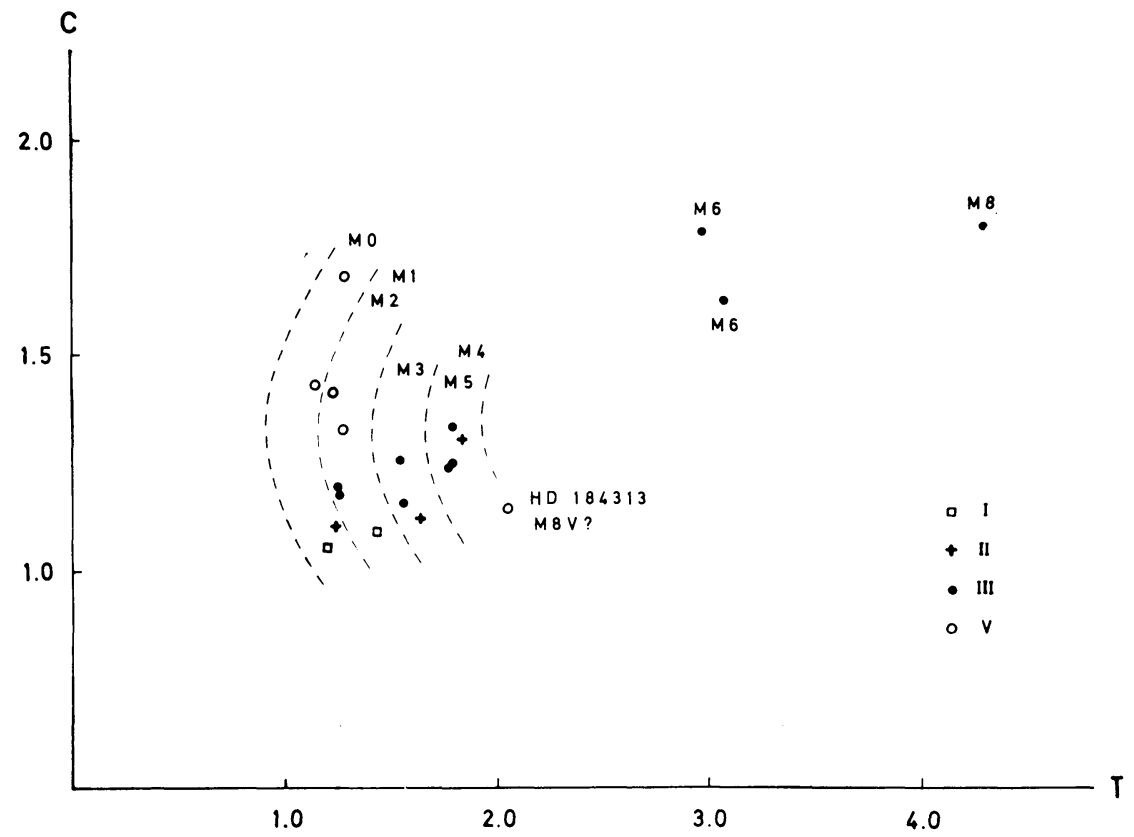

Fig. 5.

\section{References}

Elvius, T.: 1956, Stockholm Obs. Ann. 19, No. 3.

Gutiérrez-Moreno, A., Moreno, H., and Stock, J.: 1966, Publ. Dept. Astron. Univ. Chile 1, No. 2, 22. Jaschek, C., Conde, H., and de Sierra, A. C.: 1964, Obs. Astron. Univ. La Plata, Serie Astronomica 28, No. 2.

Lindblad, B. and Stenquist, E.: 1936, Stockholm Obs. Ann. 12, No. 5.

McCuskey, S. W.: 1949, Astrophys. J. 109, 426.

Mendoza, E., Moreno, H., and Stock, J.: 1968, Publ. Dept. Astron. Univ. Chile 1, No. 5, 67. Strömgren, B. and Gyldenkerne, Y.: 1955, Astrophys. J. 121, 43.

Westerlund, B.: 1951, Uppsala Astron. Obs. Ann. 3, No. 6; 1952, No. 8; 1953, No. 10. 